\title{
Niveles de actividad física en población adolescente: estudio de caso Physical activity levels in adolescents: a case study
}

\author{
Guillermo Oviedo*, Josep Sánchez*, Rubén Castro*, Mar Calvo**, Juan Carlos Sevilla***, Anna Iglesias****, Miriam Guerra* \\ *Universitat Ramon Llull, **Centro de Salud «Albera Salut», ***Fundació Salut Empordá, ****Centro de genética cardiovascular
}

\begin{abstract}
Resumen: La actividad física (AF) es fundamental en la infancia y en la adolescencia a fin de promover un desarrollo saludable. La OMS (2010), recomienda que los niños de 5-17 años deberían acumular un mínimo de 60 minutos diarios de AF moderada (3 - 6 METs) o vigorosa (> 6 METs). El objetivo general del estudio se centra en evaluar y conocer mediante la utilización de acelerometría triaxial si el tiempo e intensidad de la AF realizada diariamente por los adolescentes de un centro escolar cumplen con las recomendaciones de la OMS. Los participantes fueron 60 adolescentes pertenecientes a la escuela Empordá (Roses, España). Previamente se obtuvo el consentimiento informado de los padres y/o tutores. Los niveles de AF diarios fueron obtenidos mediante acelerometría, el índice de Masa corporal (IMC) se calculó utilizando el peso y la altura, la frecuencia cardíaca (FC) y tensión arterial (TA) se obtuvieron mediante un tensiómetro digital. Se realizó un análisis descriptivo de todas las variables y el test T de Student (p <.05) se utilizó para analizar las diferencias entre varones y mujeres. Los pricipales resultados muestran que los varones realizan AF durante más tiempo y a mayores intensidades que las mujeres. Existe un bajo porcentaje de alumnos que cumplen con las recomendaciones de la OMS. Como conclusiones, creemos muy importante la búsqueda de nuevas estrategias y métodos a fin de involucrar un mayor número de adolescentes en la práctica de $\mathrm{AF}$, como también vemos de vital inportancia las sesiones de $\mathrm{AF}$ en la escuela.
\end{abstract}

Palabra clave: actividad física, intensidad, gasto calórico, adolescentes, acelerómetro, acelerometría.

Abstract: Physical Activity (PA) is very important during childhood and adolescence in order to promote healthy development and prevent obesity. The WHO recommends 60 minutes daily of moderate-vigorous activity (e» 3 METs/day). The main objective of the study is to assess the levels of PA intensity performed daily by adolescents from a school using the triaxial accelerometry system and to know if they achieve the WHO recommendations. The sample size was 60 Spanish adolescents (14.52 \pm .854 year-old) belongs to the Empordá school (Roses, Spain). Previous informed consent was obtained from their parents and/or tutors. PA levels were obtained through accelerometry, Body Mass Index (BMI) was calculated though weight and height, heart rate (HR) and blood pressure (BP) were measured using a digital automatic blood pressure monitor. Descriptive for all data was calculated, and T-test $(\mathrm{p}<.05)$ was used to analyze the differences between boys and girls. The main result show that males performed PAduring longer time and at higher intensity than women. There is a low percentage of students who achieve the WHO recommendations. In conclusion, we believe very important to seek new strategies and methods to involve more adolescents in the practice of PA, and we believe that PA sessions at school are very important.

Key words: physical activity, intensity, energy expenditure, adolescents, accelerometry, accelerometer.

\section{Introducción}

En las últimas décadas diversos estudios científicos han demostrado que la Actividad Física (AF) regular proporciona importantes beneficios en la salud (Pate, et al., 1995; Raitakari, et al., 1997; Boreham, Twisk \& Savage, 1997; Perula de Torres, Lluch, Ruiz, Espejo, Tapia \& Mengual, 1998; Krekoukia, et al., 2007). La falta de práctica de AF está comenzando a ser un gran problema en los países desarrollados. Consecuentemente, la prevalencia de afecciones y enfermedades derivadas de la falta de actividad se están incrementando en la sociedad actual (OMS, 2010).

En el caso del sobrepeso y la obesidad, este aumento es tan alarmante en los países occidentales que algunos estudios ya hablan de este fenómeno en términos de epidemia (Banegas, 2005; Tudor Locke \& Myers, 2001). No debemos perder de vista las consecuencias que la falta de AF en la vida diaria, el sobrepeso y la obesidad tienen en la economía de los países que presentan este modo de vida, y las fuertes tasas de morbilidad y mortalidad prematuras que éstas acarrean. Por ejemplo, en los Estados Unidos de Norteamérica (EEUU), el coste directo generado por la inactividad y la obesidad consume el 9.4\% del gasto médico anual (Grundy, et al., 1999). En este mismo país, el aumento de la obesidad ha sido responsable del $27 \%$ del incremento del gasto sanitario real per cápita entre 1987 y 2001, mientras que a la diabetes se le atribuye el $38 \%$, a la hiperlipidemia el $22 \%$ y a la enfermedad cardiovascular el $41 \%$. La obesidad por sí misma, es decir, como factor de riesgo independiente, sería responsable del $12 \%$ del aumento del gasto sanitario de este período (Thorpe, Florence, Howard \& Joski, 2004).

Fecha recepción: 17-06-12 - Fecha envío revisores: 17-06-12 - Fecha de aceptación: 21-07-12 Correspondencia: Guillermo Ruben Oviedo

Carrer Císter 34,

08022, Barcelon

E-mail: GuillermoRubenO@blanquerna.url.edu
En la Unión Europea se producen más de 13 millones de muertes anuales relacionadas con el exceso de peso (Banegas, López-García, Gutiérrez-Fisac, Guallar-Castillón \& Rodríguez-Artalejo, 2003), y el número de niños que padecen sobrepeso y obesidad infantil se está incrementando en, aproximadamente, 1.3 millones al año (JacksonLeach \& Lobstein, 2006). Según el estudio en Kid (Serra, et al., 2003; Serra, Aranceta, Pérez, Ribas \& Delgado, 2006), realizado en España, la prevalencia de la obesidad es casi del $14 \%$ entre los menores de 18 años; y se conoce que el coste directo generado por la inactividad y la obesidad ronda ya el 7\% del gasto médico anual (Vázquez \& López, 2003).

$\mathrm{La} \mathrm{AF}$ es fundamental en la infancia y en la adolescencia a fin de promover un desarrollo saludable. Su práctica, siempre y cuando sea apropiada a la edad de los participantes, tiene efectos beneficiosos en el desarrollo motor, cognitivo, afectivo, social, y prácticamente en todos los sistemas (Armstrong, et al., 2007).

Es a lo largo de la infancia y la adolescencia cuando se instauran muchas pautas de comportamiento que van a tener una influencia poderosa sobre la salud en la vida adulta (National Center for Chronic Disease Prevention and Health Promotion, Centres for Disease Control and Prevention, 1997; Perula de Torres, et al., 1998; Janz, Dawson \& Mahoney, 2000).

Estudios previos donde se utilizaron acelerómetros para la evaluación de los niveles de $\mathrm{AF}$ en niños y adolescentes de 9 a 16 años en Europa y España, se detectaron mayores niveles de $\mathrm{AF}$ en varones (Riddoch, et al., 2004; Martínez-Gómez, Welk, Calle, Marcos \& Veiga, 2009).

La evidencia científica disponible con respecto al grupo de edades de 5 a 17 años respalda la conclusión de que la $\mathrm{AF}$ reporta en general beneficios fundamentales para la salud de niños y jóvenes. Esta conclusión está basada en los resultados de varios estudios que han observado que el aumento de $\mathrm{AF}$ estaba asociada a unos parámetros de salud más favorables, y de trabajos experimentales que han demostrado cierta asociación entre las intervenciones de AF y la mejora de los indicadores 
de salud. Algunos de los beneficios documentados son: mejora de la forma física (tanto de las funciones cardiorrespiratorias como de la fuerza muscular), reducción de la grasa corporal, perfil favorable de riesgo de enfermedades cardiovasculares y metabólicas, mayor salud ósea, y menor presencia de síntomas de depresión (Janssen, 2007; Physical Activity Guidelines Advisory Committee, 2008).

Los beneficios que la AF produce sobre el sistema músculoesquelético, cardio vascular, tensión arterial y porcentaje graso son diversos. Así, Strong et al. (2005) refieren que la AF aeróbica de moderada intensidad, de 30 a 60 minutos de duración, 3 o 7 días/semana reducen la grasa visceral y total en niños/as y adolescentes con sobrepeso, aumenta el c-HDL y disminuye los triglicéridos, mejora la forma física y el trabajo muscular 2 o 3 días/semana (con descanso entre las sesiones) mejora la fuerza y la resistencia muscular en niños/as y adolescentes.

Asimismo, aunque de forma menos consistente, Strong et al. (2005) concluyen que la práctica de $\mathrm{AF}$ por parte de de niños y adolescentes con peso normal mejora los niveles de lípidos y lipoproteínas en sangre, disminuye la adiposidad, la presión arterial en jóvenes normotensos, y mejora la autoestima, la ansiedad, los síntomas de depresión y el rendimiento académico.

La OMS (2010), con objeto de mejorar las funciones cardiorespiratorias, la buena forma muscular, la salud ósea y los biomarcadores cardiovasculares y metabólicos, recomienda:

- Los niños de 5-17 años deberían acumular un mínimo de 60 minutos diarios de AF moderada (3 - 6 METs) o vigorosa (> $6 \mathrm{METs}$ ).

- La AF durante más de 60 minutos reporta beneficios adicionales para la salud.

- La AF diaria debería ser, en su mayor parte, aeróbica. Convendría incorporar actividades vigorosas, en particular para fortalecer los músculos y los huesos, como mínimo tres veces a la semana.

La valoración precisa y confiable de la actividad física es necesaria en cualquier estudio en donde la $\mathrm{AF}$ sea la intervención experimental o una medida de los resultados (Rowlands, Powell, Humphries \& Eston, 2006; Rowlands \& Eston, 2007).

El uso de censores que proveen medidas objetivas del movimiento -los cuales ofrecen estimaciones más precisas de la actividad física- ha sido demostrado que es válido ya que éstos son válidos (Ekelund, et al., 2001), confiables (Metcalf, Curnow, Evans, Voss \& Wilkin, 2002) y prácticos de utilizar en grandes estudios (Pate, et al., 2002).

No existen estudios previos en la Comunidad Autónoma de Cataluña donde se utilizase acelerometría triaxial para evaluar los niveles de AF de adolescentes. Por esta razón y teniendo en cuenta las consideraciones anteriores, nos planteamos como objetivo general del estudio conocer mediante la utilización de acelerometría triaxial si el tiempo e intensidad de la $\mathrm{AF}$ realizada diariamente por los adolescentes de un centro escolar de la ciudad de Roses (España) cumple con las recomendaciones que la OMS realiza en este sentido.

También nos planteamos los siguientes objetivos específicos:

- Determinar el tiempo total de AF e» a 3 METs que realizan los alumnos, según el sexo y edades.

-Cuantificar el gasto energético según sexo y edades.

-Evaluar la frecuencia cardíaca de reposo (FC), la tensión arterial sistólica/diastólica (TAS/TAD) y el porcentaje de masa grasa (PMG).

\section{Metodología}

\subsection{Participantes}

En nuestro estudio participaron 60 alumnos, tanto varones como mujeres, de la escuela superior obligatoria (ESO) concertada Empordá de la ciudad de Roses, provincia de Girona. Las edades de los participantes se encuentran entre los 13 y 16 años.

\subsection{Instrumentos}

Acelerómetro triaxial Actigraph GT3X (ActiGraphTM, LLC, Fort Walton Beach, FL, USA).

Báscula romana, precisión 100g, ASIMED MB 201T. (Asimed S.A., Barcelona, Spain).
Tallímetro rígido, precisión 0,1cm, ASIMED MB 201T, de lectura directa. (Asimed, Barcelona, Spain).

Tensiómetro digital OMRON M4-I (HEM 752/E, OMRON

HEALTHCARE Co., Ltd., Kyoto, Japan).

Plicómetro Holtein. (Holtain Ltd., Walles, UK).

\subsection{Procedimientos}

En el presente estudio todos los alumnos fueron previamente informados sobre los protocolos de evaluación y se obtuvo el consentimiento informado de padres y/o tutores. El estudio tuvo la aprobación del comité de ética del centro de investigación.

$\mathrm{Al}$ iniciar el trabajo se obtuvo la edad de los participantes y se midieron la talla y peso utilizando procedimientos estandarizados (Aragonés, 1989). La FC, TAS, TAD se obtuvo utilizando un tensiómetro digital, con el evaluado en reposo y sentado.

La medición de los pliegues grasos se realizó sobre el lado derecho de los alumnos en los siguientes lugares: tríceps, subescapular, suprailíaco y abdominal. Se determinó la densidad corporal (Jackson \& Pollock, 1978) y con esta el porcentaje de masa grasa (Siri, 1961).

\begin{tabular}{|c|c|c|} 
Tabla 1. Dis tri bución de los su jetos según edad y sexo. \\
\hline Edad (años) & Chi cos & Chicas \\
\hline 13 & 5 & 1 \\
14 & 15 & 10 \\
15 & 10 & 11 \\
16 & 3 & 5 \\
\hline
\end{tabular}

Tabla 2. Análisis des criptivo según sexo, edad, peso, talla e IMC, FC, TAS y TAD de los 60 participantes.

\begin{tabular}{|l|c|c|c|c|}
\hline \multirow{2}{*}{} & \multicolumn{2}{|c|}{ Chicos } & \multicolumn{2}{c|}{ Chicas } \\
\cline { 2 - 5 } & Media & Desviación típica & Med ia & Desvi ación típica \\
\hline Edad (años) & 14,33 & 0,85 & 14,74 & 0,81 \\
\hline Peso $(\mathrm{kg})$ & 61,45 & 10,64 & 56,17 & 10,85 \\
\hline Talla $(\mathrm{m})$ & $1,68^{*}$ & 0,08 & 1,64 & 0,05 \\
\hline IMC $\left(\mathrm{kg} / \mathrm{m}^{2}\right)$ & 21,76 & 3,06 & 20,91 & 3,31 \\
\hline PMG $(\%)$ & 21,13 & 4,16 & $25,49^{*}$ & 2,57 \\
\hline FC (lat $x$ min) & 71,76 & 13,65 & 73,83 & 10,23 \\
\hline TAS $(\mathrm{mmHg})$ & $124,79^{*}$ & 10,78 & 117,85 & 10,83 \\
\hline TAD $(\mathrm{mm} H \mathrm{mg})$ & 65,24 & 7,72 & 65,70 & 7,20 \\
\hline
\end{tabular}

\section{*Diferencias significativas ( $\mathrm{p}<.005)$}

Para la medición del gasto energético e intensidades de AF realizada los participantes utilizaron acelerómetros triaxiales GT3X. Estos monitores de actividad miden aceleraciones sobre los tres planos anatómicos del cuerpo (sagital, frontal y transversal) y ofrece cuentas del movimiento como un vector resultante de las magnitudes de cada uno de los tres ejes.

Se evaluaron tres días. El primer día correspondía al día de clases de Educación Física, el segundo era un día donde no había clases de Educación Física y el tercero fue el sábado. Los datos fueron tomados por los acelerómetros entre las $9 \mathrm{hs}$ de la mañana hasta las $23: 59 \mathrm{hs}$ del mismo dí. Los acelerómetros fueron colocados en el lado derecho a nivel de la cadera.

Los acelerómetros fueron calibrados en épocas de 60", con funciones de podómetro e inclinómetro, se utlizó el vector magnitud de los tres ejes para detectar los períodos de utilización o no, excluyéndose los períodos donde se contabilizaron períodos de 10 minutos de ceros contínuos. Sólo fueron incluidos los datos donde los acelerómetros obtuvieron información durante 10 horas diarias, como mínimo.

Para determinar los niveles de AF se utilizó el vector magnitud de los tres ejes (Sasaki, John \& Freedson, 2011). Luego se clasificó la intensidad (MET) según el modelo propuesto por Pate et al. (Ainsworth, et al., 2000; Pate, et al., 1995).

La obtención del gasto energético (Kcal) se determinó por medio de la ecuación de Freedson (Freedson, Melanson \& Sirard, 1998) y el Work Energy Theorem $(1 \mathrm{kcal}=$ Cuentas $\times .0000191 \times$ peso de la persona en $\mathrm{kg}$ ).

\subsection{Análisis estadístico de los datos}

Para la descarga y posterior análisis de los datos provenientes de los acelerómetros, se utilizó el software ActiLife5 v5.7.4. 
Se utilizó el paquete SPSS, versión 19.0, para realizar un análisis descriptivo e inferencial de las variables estudiadas. Se utilizó la prueba estadística $\mathrm{X}^{2}$ (Chi-cuadrado de Pearson) para determinar las asociaciones entre sexo y cumplimiento de las recomendaciones OMS sobre la AF.

También se realizó el test t-Student entre sexo y tiempo medio de $\mathrm{AF}$ e» 3Mets diario y total, gasto calórico total y diario, talla, PMG y TAS. En cada caso se trabajó con un nivel de confianza del $95 \%$.

\section{Resultados}

Las características de los participantes del estudio se presentan en la tabla 2. Los chicos fueron significativamente más altos que las mujeres $(\mathrm{t}=2.46 ; \mathrm{p}=.017)$ y presentaron valores significativamente más altos de TAS $(t=2.47 ; p=.016)$. Las chicas presentaron valores significativamente mayores de PMG $(\mathrm{t}=4.60 ; \mathrm{p}<.001)$. Con respecto al peso, IMC, FC y TAD no se encontraron diferencias significativas entre sexos.

3.1. Actividad Física realizada por los alumnos según sexo

La figura 1 nos describe la media de minutos de AF realizada a intensidades e» 3 METs, cada uno de los días evaluados.

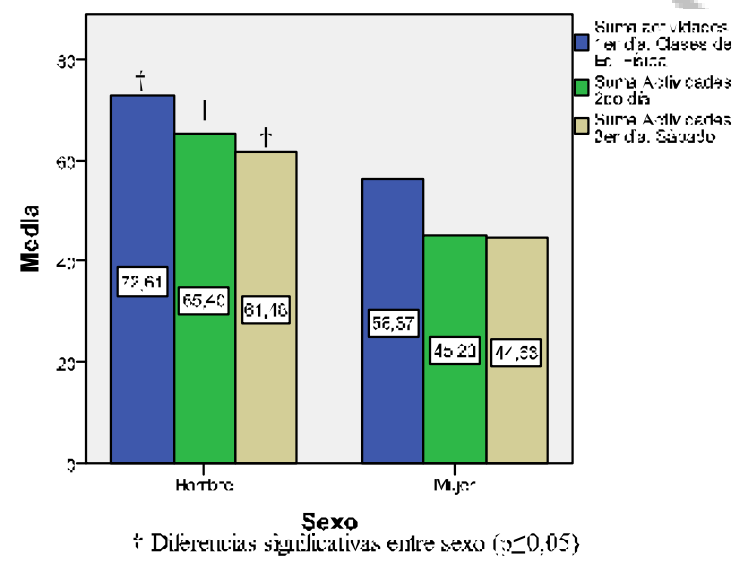

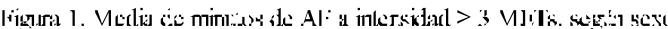

El 31.7\% del total de adolescentes avaluados, realizaron al menos 60 minutos de AF e» a 3 METs los tres días. De ellos, el 45.5\% fueron los varones, y el $14.84 \%$ fueron las mujeres lo hizo. La asociación entre sexo masculino y el cumplimiento de las recomendaciones de $\mathrm{AF}$ de la OMS durante los días evaluados fue significativa $\left(\mathrm{X}^{2}=6.44 ; \mathrm{p}=.011\right)$.

Durante el primer día evaluado, que correspondía con el día en que realizaban la sesión de AF, el $66.7 \%$ de los varones y el $40.7 \%$ de las mujeres cumplían con las recomendaciones OMS sobre AF.

El segundo día evaluado, el $53.3 \%$ de los varones y el $23.1 \%$ de las mujeres cumplieron con las recomendaciones de la OMS sobre AF.

El tercer día (sábado), sólo el 51.5\% de varones y el 14.8\% de mujeres cumplieron con las recomendaciones.

Se encontraron asociaciones significativas entre el sexo masculino y el cumplimiento de las recomendaciones de la OMS sobre la realización de $\mathrm{AF}$ durante los tres días (primer día: $\mathrm{X}^{2}=4.03 ; \mathrm{p}=.045$; segundo día $\mathrm{X}^{2}=5.34 ; \mathrm{p}=.021$; tercer día: $\mathrm{X}^{2}=8.79 ; \mathrm{p}=.003$ ).

El tiempo total de $\mathrm{AF}$ e» a 3 METs realizada durante los días evaluados fue $193,54 \pm 68,12$ minutos en los chicos y $144.55 \pm 61.58$ minutos en las chicas. Aplicando el test t-Student se detecta que las diferencias entre sexos son estadísticamente significativas $(\mathrm{t}=2.89$; $\mathrm{p}=.005)$. La media de $\mathrm{AF}$ e» a 3 METs realizada por los varones fue de $64.51 \pm 22.70$ minutos y la de las mujeres fue $48.18 \pm 20.52$. Las diferencias entre sexo son significativas $(\mathrm{t}=2.89 ; \mathrm{p}=.005)$.

Durante el primer día evaluado los chicos realizan $72.61 \pm 30.7$ minutos de $\mathrm{AF}$ e» a 3 METs y las chicas realizan $56.37 \pm 25.75$ minutos de AF e» a 3 METs. Aplicando el test t-Student, se detecta que la diferencia entre sexos es significativa $(\mathrm{t}=2.18 ; \mathrm{p}=.033)$.
El segundo día evaluado los chicos realizan $65.4 \pm 31.63$ minutos de $\mathrm{AF}$ e» a 3 METs mientras que las chicas realizan $45.23 \pm 24.87$ minutos de $\mathrm{AF}$ e» a 3 METs. Esta diferencia entre sexos es significativa ( $\mathrm{t}=2.63$; $\mathrm{p}=.011$ ).

El día sábado, que fue el tercer día evaluado, los chicos realizan $61.48 \pm 25.34$ minutos de $\mathrm{AF}$ e» a 3 METs y las chicas realizan $44.62 \pm 16.55$ minutos de $\mathrm{AF}$ e» a 3 METs. Estas diferencias son significativas ( $\mathrm{t}=2.97 ; \mathrm{p}=.004)$.

El tiempo empleado por los varones en $\mathrm{AF}$ e» a 3 METs durante el primer día es significativamente mayor que el tercer día $(\mathrm{t}=2.6$; $\mathrm{pd})$.05) al igual que el segundo día con respecto al tercero $(t=2.7 ; p=.01)$. En el caso de las mujeres, existen diferencias significativas entre el primer día evaluado y el segundo $(\mathrm{t}=2.68 ; \mathrm{p}=.013)$ como también entre el primer día y el tercero $(\mathrm{t}=3.66 ; \mathrm{p}=.001)$.

3.2. Actividad Física realizada por los alumnos según edades

La muestra se agrupó en dos franjas, que corresponden al primer ciclo ESO (13-14años) y segundo ciclo ESO (15-16 años).

El tiempo total de $\mathrm{AFe}$ » 3 METs realizada por los alumnos de 1314 años fue de $173.74 \pm 74.42$ minutos y los de $15-16$ años $169.10 \pm 64.43$ minutos. En la figura 2 se observan las medias de $\mathrm{AFe}$ » a 3 METs según edades.

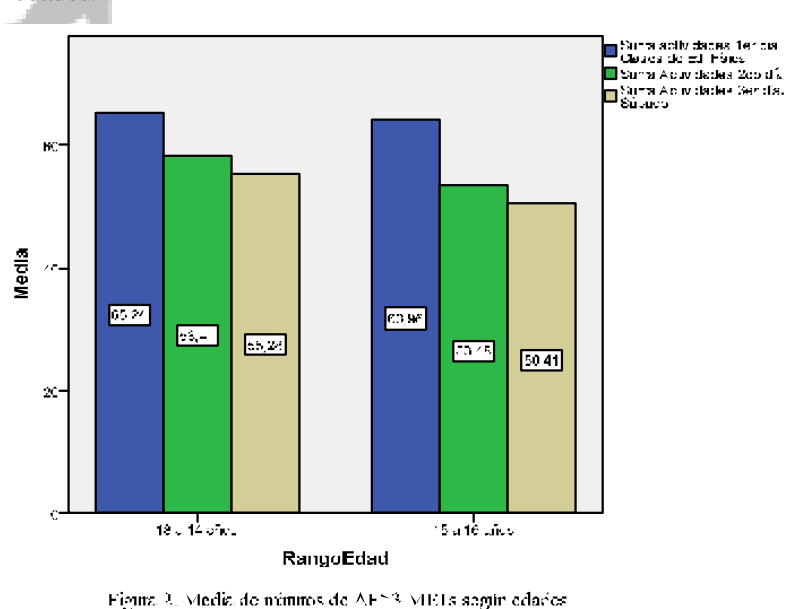

$\mathrm{Al}$ analizar el tiempo de $\mathrm{AF}$ e» a 3 METs cada día evaluado según edades, se encontróque los alumnos de 13 a 14 años realizan $64.16 \pm 28.56$ minutos el primer día y los de 15 a 16 años realizan $66.52 \pm 30.91$ minutos; el segundo día los de 13 a 14 años realizan $58.41 \pm 32.93$ minutos y los de 15 a 16 años 53.48 \pm 27.34 minutos; el tercer día los alumnos de 13 a 14 años realizaron $54.93 \pm 23.82$ minutos mientras que los de 15 a 16 años realizaron $52.79 \pm 22.99$ minutos.

$\mathrm{Al}$ analizar las diferencias del tiempo total y diario en la realización de $\mathrm{AF}$ e» a 3 METs por edades, no se encontraron diferencias significativas.

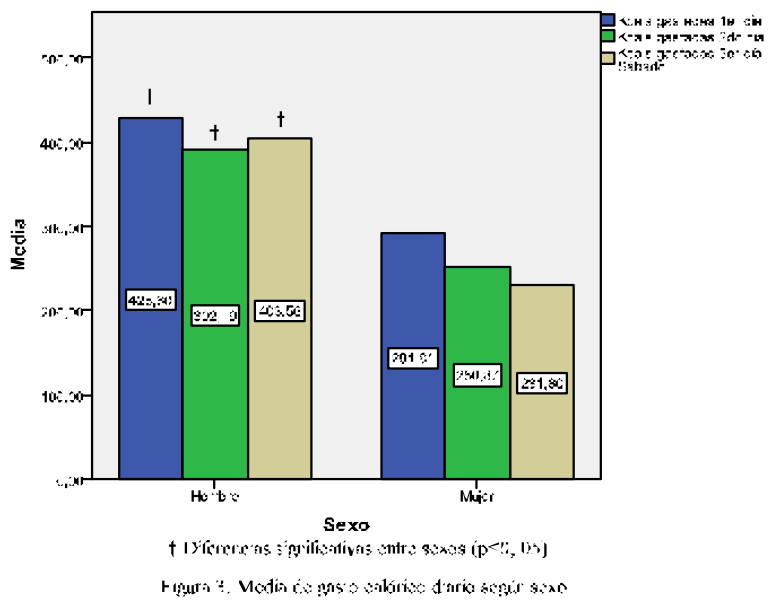




\subsection{Gasto calórico de los alumnos según sexo}

En la figura 3 podemos ver el gasto calórico diario medio de los alumnos según sexo.

El gasto calórico total fue $1188.68 \pm 514.88$ kcals. en los chicos y $765.18 \pm 387.04$ kcals. en las mujeres. Las diferencias fueron significativas $(\mathrm{t}=3.53 ; \mathrm{p}=.001)$.

Durante el primer día evaluado, los chicos realizaron un gasto calórico medio de $428.59 \pm 214.60$ kcals. y las chicas $291.80 \pm 144.04$. Las diferencias fueron significativas $(\mathrm{t}=2.82 ; \mathrm{p}=.006)$.

El segundo día los chicos realizaron un gasto calórico medio de $392.18 \pm 217.11$ kcals. y las chicas $250.87 \pm 146.48$. Las diferencias fueron significativas $(\mathrm{t}=2.80 ; \mathrm{p}=.007)$.

El tercer día evaluado los chicos realizaron un gasto calórico medio de 403.55 \pm 246.96 kcals. y las chicas $231.79 \pm 127.44$. Las diferencias fueron significativas $(\mathrm{t}=3.27 ; \mathrm{p}=.002)$.

\subsection{Gasto calórico de los alumnos según edades}

En la figura 4 se verel gasto calórico medio diario según edades.

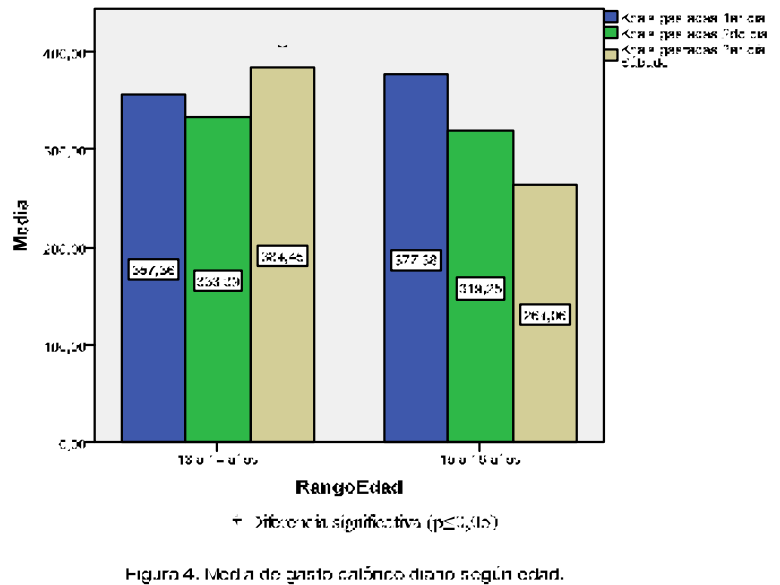

El gasto calórico total fue de $1053.70 \pm 559.88$ kcals. en los alumnos de 13 a 14 años y $938.68 \pm 440.74$ kcals. en los de 15 a 16 años.

El gasto calórico de los alumnos de 13 a 14 años durante el primer día fue de $357.36 \pm 178.58 \mathrm{kcals}$. y el de los alumnos de 15 a 16 años fue de $377.38 \pm 217.80$.

Durante el segundo día los alumnos de 13 a 14 años realizaron un gasto calórico de $333.39 \pm 203.36$ kcals. y los de 15 a 16 años $319.25 \pm 198.14$ kcals.

Durante el tercer día los alumnos de 13 a 14 años realizaron un gasto calórico de $384.45 \pm 243.90$ kcals. y los de 15 a 16 años $264.06 \pm 170.11$ $\mathrm{kcals}$. En este día las diferencias fueron significativas $(\mathrm{t}=2.20 ; \mathrm{p}=.032)$.

\section{Discusión}

Los resultados expuestos anteriormente se refieren a los estudiantes de la Escuela Empordá de la ciudad de Roses, provincia de Girona. Hasta donde se conozca, este es el primer estudio en el cual se evalúa los niveles de AF e» a 3 METs en Cataluña, utilizando como método de evaluación la acelerometría triaxial.

Los principales resultados que hemos encontrado en nuestro estudio son la asociación significativa del sexo masculino con el cumplimiento de las recomendaciones de realizar $\mathrm{AF}$ e» a 3 METs durante al menos 60 minutos todos los días. Con respecto a esta recomendación, el $45.5 \%$ del total de varones y sólo el $14.84 \%$ de las mujeres la cumplen. Al unir los dos sexos, obtenemos que el $31,7 \%$ de los alumnos cumplen las recomendaciones de la OMS. En un estudio anterior realizado en la ciudad de Huesca, si bien se evaluaron siete días, se determinó que sólo el $7.54 \%$ de los chicos y el $3.44 \%$ de las chicas cumplían las recomendaciones (Abarca-Sos, Zaragoza, Generelo \& Julián, 2010). En otro estudio realizado en la región de Madrid donde se evaluaron siete días, el $82.2 \%$ de los chicos y el $60.7 \%$ de las chicas cumplieron con las recomendaciones todos los días evaluados (Martínez-Gómez, et al., 2009).
También al analizar por separado cada uno de los tres días en los que los alumnos llevaron los acelerómetros, encontramos asociaciones significativas entre el sexo masculino y el cumplimiento de las recomendaciones de la OMS. Durante el primer, segundo y tercer día el 66.7\%, $53.3 \%$ y $51.5 \%$ de los varones, respectivamente, cumplen las recomendaciones. Por su lado, sólo el $40.7 \%, 23.1 \%$ y $14.8 \%$ de las mujeres lo hacen el primer, segundo y tercer día respectivamente. En otros estudios donde se evaluó a los participantes entre siete y tres días, también se observa un menor nivel de $\mathrm{AF}$ en las mujeres (Riddoch, et al., 2004; Martínez-Gómez, et al., 2009).

El tiempo diario medio durante el cual los alumnos evaluados en nuestro estudio desarrollaron AF e» a 3 METs fue de 64.51 \pm 22.7 minutos en los varones y de $48.18 \pm 20.52$ minutos en las mujeres. Estos valores son menores a los encontrados en estudios realizados con adolescentes en la región de Madrid, donde los varones realizan una media de AF e» a 3 METs de $85.09 \pm 29.22$ minutos y $62.70 \pm 19.64$ minutos en mujeres (Martínez-Gómez, et al., 2009) así como en los resultados que se observan en adolescentes de Dinamarca, Portugal, Estonia y Noruega, donde los varones desarrollan una media de 77, 110, 110 y 92 minutos de $\mathrm{AF}$ e» a 3 METs diaria respectivamente y las mujeres 60, 80, 74 y 82 minutos de $\mathrm{AF}$ e» a 3 METs respectivamente ( Riddoch, et al., 2004). Valores menores a los nuestros fueron los encontrados en un estudio desarrollado en Estados Unidos de Norteamérica, donde los adolescentes de 12 a 19 años varones sólo alcanzaban entre 45.3 y 32.7 minutos de $\mathrm{AF}$ e» a 3 METs respectivamente y las mujeres 24.6 a 19.6 minutos de $\mathrm{AF}$ e» a 3 METs respectivamente (Troiano, Berrigan, Dodd, Masse, Tilert \& McDowell, 2007).

Consideramos importantes los resultados encontrados donde podemos observar que los alumnos tanto varones como mujeres, realizan un mayor número de minutos de $\mathrm{AF}$ e» a 3 METs durante los días escolares, siendo significativas las diferencias entre el los dos días escolares y el fin de semana, en el caso de los varones, y entre el primer día evaluado (donde hubo sesiones de $\mathrm{AF}$ en el colegio) y los otros dos días en el caso de las mujeres. Estos resultados concuerdan con estudios anteriores, donde se indica que los adolescentes realizan más AF durante los días escolares que los fines de semana (Nader, Bradley, Houts, McRitchie \& O'Brien, 2008; Nilsson, et al., 2009; Abarca-Sos, et al., 2010).

Con respecto al gasto calórico encontramos que los varones presentan un gasto calórico mayor total, así como en cada uno de los tres días evaluados. En un estudio donde se evaluaron el gasto calórico a 20 niños y adolescentes españoles durante 24 horas un día escolar mediante acelerometría triaxial (Rodríguez, Béghin, Michaud, Moreno, Turk \& Gottrand, 2002) se observó que tenían un mayor gasto calórico que en nuestro estudio.

Se deberían tener en cuenta una serie de limitaciones de este estudio. En primer lugar, nuestro estudio fue realizado con acelerómetros ActiGraph y actualmente no existe un único consenso sobre cuáles son los puntos de corte para evaluar la intensidad de la AF que realizan los adolescentes (Freedson, Pober \& Janz, 2005). Estos acelerómetros tampoco fueron diseñados para utilizarse en el medio acuático o en la ducha, por lo que las actividades en el mar o en la piscina no quedan registradas en los acelerómetros. El acelerómetro ActiGraph GT3X es triaxial por lo que pueden llegar a existir errores en la comparación con acelerómetros que detectan sólo los movimientos verticales (Sasaki, et al., 2011). También las épocas de 60 segundos utilizadas en nuestro estudio podrían subestimar las actividades vigorosas y de corta duración que realizan los niños y adolescentes (Freedson, et al., 2005; Guinhouya, Hubert, Soubrier, Vilhelm, Lemdani \& Durocher, 2006).

\section{Conclusiones}

Los resultados de este estudio nos indican que los adolescentes dedican poco tiempo a la realización de AF e» a 3 METs. En particular, las mujeres no alcanzan las mínimas recomendaciones sobre la práctica de $\mathrm{AF}$ en ninguno de los días evaluados. 
Esto nos hace pensar que las propuestas de $\mathrm{AF}$ que a estas edades existen, podrían presentar una carga deportiva que resulta más atractiva a los varones que a las mujeres, por lo que sería necesario realizar nuevas propuestas y planteamientos pedagógicos a fin de lograr que las mujeres incrementen el tiempo de práctica de $\mathrm{AF}$ e» a 3 METs.

Creemos que el estudio demuestra que la sesión de actividad física en la escuela es muy importante ya que es el día donde los adolescentes presentaron un mayor número de minutos de $\mathrm{AF}$ e» a 3 METs, tanto varones como mujeres. El incremento de horas lectivas de actividad física en las escuelas serviría en gran medida a incrementar la $\mathrm{AF}$ practicada por los alumnos. Esto es de vital importancia, ya que los hábitos sanos y saludables que los niños y adolescentes aprendan, les permitirá incrementar sus posibilidades de ser adultos saludables.

Creemos necesario realizar nuevas investigaciones a fin de incrementar la muestra de alumnos participantes y poder obtener datos con un mayor valor estadístico, incluyendo otras franjas de edad así como escuelas de ámbito urbano, suburbano y rural.

\section{Referencias}

Abarca-Sos, A., Zaragoza, J., Generelo, E., \& Julián, J. A. (2010). Comportamientos sedentarios y patrones de actividad física en adolescentes. Revista Internacional de Medicina y Ciencias de la Actividad Física y del Deporte, 40(39), 410-427.

Ainsworth, B., Haskell, W., Whitt, M., Irwin, M. L., Swartz, A. M., Strath, S. J Leon, A. S. (2000). Compendium of physical activities: an update of activity codes and MET intensities. Medicine and Science in Sports and Exercise, 32(9), Suppl.,pp. S498-S516.

Aragonés, M. (1.989) Cineantropometría: puntos anatómicos y técnicas de medición. Jornadas de estandarización del Grupo Español de cineantropometría. Grec. Car. Sant Cugat.

Armstrong, N., \& Welsman, J. R. (2007). The Contribution of Physical Activity and Sedentary Behaviours to the Growth and Development of Children and Adolescents. Implications for Overweight and Obesity. Sports Medicine, 37(6), 1067-1086.

Banegas, J. R., López-García, E., Gutiérrez-Fisac, J. L., Guallar-Castillón, P., \& Rodríguez-Artalejo, F. (2003). A simple estimate of mortality attributable to excess weight in the European Union. European Journal of Clinical Nutrition, 57(2), 201-208.

Banegas, J. R. (2005). Epidemiología de la hipertensión arterial en España. Situación actual y perspectivas. Hipertensión, 22, 353-362.

Boreham, C. A., Twisk, J., \& Savage, M. J. (1997). Physical activity, sports participation, and risk factors in adolescents. Medicine and Science in Sports and Exercise, 29, 788-793.

Ekelund, U., Sjöström, M., Yngve, A., Poortvliet, E., Nilsson, A., Froberg, K.... Westerterp, K. (2001). Physical activity assessed by activity monitor and doubly labeled water in children. Medicine and Science in Sports and Exercise, 33(2), 275-81.

Freedson, P. S., Melanson, E., \& Sirard, J. (1998) Calibration of the Computer Science and Applications, Inc. accelerometer. Medicine and Sciences in Sports Exercise, 30(5), 777-781.

Freedson, P., Pober, D., \& Janz, K. F. (2005) Calibration of accelerometer output for children. Medicine and Science in Sports and Exercise, 37(11 Suppl.), 523 530

Guinhouya, C. B., Hubert, H., Soubrier, S., Vilhelm, C., Lemdani, M., \& Durocher, A. (2006). Moderate to Vigorous Physical activity among children: Discrepances in accelerometry - Based cut-off points. Obesity, 14(5), 774-8.

Grundy, S. M., Blackburn, G, Higgins, M., Lauer, R., Perri, M. G, \& Ryan, D. (1999) Physical activity in the prevention and treatment of obesity and its comorbidities: evidence report of independent panel to assess the role of physical activity in the treatment of obesity and its comorbidities. Medicine and Science in Sports and Exercise, 31(11), 1493-1500.

Jackson-Leach, R., \& Lobstein, T. (2006). Estimated burden of paediatric obesity and co-morbidities in Europe. Part 1. The increase in the prevalence of child obesity in Europe is itself increasing. International Journal of Pediatric Obesity, 1(1), 26-32.

Jackson, A. S., \& Pollock, M. L. (1978). Generalized equations for predicting body density of men. British Journal of Nutrition, 40, 497-504.

Janssen, I. (2007). Physical activity guidelines for children and youth. Applied Physiology Nutrition and Metabolism, 32, S109-S121.

Janz, K. F., Dawson, J. D., \& Mahoney, L. T. (2000). Tracking physical fitness and physical activity from childhood to adolescence: the muscatine study. Medicine and Science in Sports and Exercise, 32(7), 1250-1257.

Krekoukia, M., Nassis, G P., Psarra, G, Skenderi, K., Chrousos, G. P., \& Sidosis, L. S. (2007). Elevated total and central adiposity and low physical activity are associated with insulin resistance in children. Metabolism, 56, 206-213.
Martínez-Gómez, D., Welk, G J., Calle, M. E., Marcos, A., \& Veiga, O. L.; AFINOS Study Group (2009). Preliminary evidence of physical activity levels measured by accelerometer in Spanish adolescents: the AFINOS Study. Nutrición Hospitalaria, 24 (2), 226-232.

Metcalf, B. S., Curnow, J. S., Evans, C., Voss, L. D., \& Wilkin, T. J. (2002) Technical reliability of the CSA activity monitor: The EarlyBird Study. Medicine and Sciences in Sports and Exercise, 34(9), 1533-1537.

National Center for Chronic Disease Prevention and Health Promotions, Centers for Disease Control and Prevention. (1997). Guidelines for school and community programs to promote lifelong physical activity among young people. The Journal of School Health, 67(6), 202-219.

Nader, P. R., Bradley, R. H., Houts, R. M., McRitchie, S. L., \& O’Brien, M. (2008). Moderate to vigorous physical activity from ages 9 to 15 . Journal of the American Medical Association, 300(3), 295-305.

Nilsson, A., Anderssen, S. A., Andersen, L. B., Froberg, K., Riddoch, C., Sardinha, L. B., \& Ekelund, U. (2009) Between- and within-day variability in physical activity and inactivity in 9 - and 15 -year-old European children. Scandinavian Journal of Medicine \& Science in Sports, 19(1), 10-8.

Organización Mundial de la Salud. (2010). Recomendaciones mundiales sobre actividad física para la salud. Ginebra: WHO.

Pate, R. R., Freedson, P. S., Sallis, J. F., Taylor, W. C., Sirard, J., Trost, S. G, \& Dowda, M. (2002) Compliance with physical activity guidelines: prevalence in a population of children and youth. Annals of Epidemiology, 12(5), 303-308.

Pate, R. R., Pratt, M., Blair, S. N., Haskell, W. L., Macera, C. A., Bouchard, C. King, A. C. (1995). Physical activity and public health: a recommendation from the Centers for Disease Control and Prevention and the American College of Sports Medicine. JAMA, 273, 402-407.

Perula de Torres, L. A., Lluch, C., Ruiz, R., Espejo, J., Tapia, G, \& Mengual, P. (1998). Prevalencia de actividad física y su relación con variables sociodemográficas y ciertos estilos de vida en escolares cordobeses. Revista Española de Salud Pública, 72, 233-244.

Physical Activity Guidelines Advisory Committee (PAGAC). (2008). Physical Activity Guidelines Advisory Committee Report, 2008. Washington, DC, US Department of Health and Human Services.

Raitakari, O. T., Taimela, S., Porkka, K. V. K., Telama, R., Välimäki, I., Akerblom H. K., \& Viikari, J. S. A. (1997). Associations between physical activity and risk factors for coronary heart disease: the cardiovascular risk in young finns study. Medicine and Science in Sports and Exercise, 29, 1055-1061.

Riddoch, C. J., Bo Andersen, L., Wedderkopp, N., Harro, M., Klasson-Heggeb $\varnothing$, L., Sardinha, L. B.... Ekelund, U. (2004). Physical activity levels and pattern of 9- and 15-yr-old European children. Medicine and Sciences in Sports and Exercise, 36(1), 86-92.

Rodríguez, G, Béghin, L., Michaud, L., Moreno, L. A., Turk, D., \& Gottrand, F. (2002) Comparison of the TriTrac-R3D accelerometer and a self-report activity diary with heart-rate monitoring for the assessment of energy expenditure in children. British Journal of Nutrition, 87, 623-631.

Rowlands, A. V., \& Eston, R. G. (2007). The measurement and interpretation of children's physical activity. Journal of Sports Sciences and Medicine, 6, 270276

Rowlands, A. V., Powell, S. M., Humphries, R., \& Eston, R. G (2006). The effect of accelerometer epoch on physical activity output measures. Journal of Exercise Sciences and Fitness, 4(1), 52-58.

Sasaki, J. E., John, D., \& Freedson, P.S. (2011). Validation and comparison of ActiGraph activity monitors. Journal of Science and Medicine in Sport, 14 411-416.

Serra, L., Ribas, L., Aranceta, J., Pérez, C., Saavedra, P., \& Pena, L. (2003) Obesidad infantil y juvenil en España. Resultados del Estudio enKid (19982000). Med Clin (Barc), 121(19), 725-732.

Serra, L., Aranceta, J., Pérez, C., Ribas Barba, L., \& Delgado Rubio, A. (2006) Prevalence and determinants of obesity in Spanish children and young people. The British Journal of Nutrition, 96, Suppl.1, pp. S67-S72.

Siri, W. E. (1961). Body composition from fluid spaces and density: analysis of methods. En J. Brozek, A. Henschel (Eds.), Techniques for Measure of body composition (pp. 223-244). Washington: Acad Sci Nat Res Council.

Strong, W. B., Malina, R. M., Blimkie, C. J., Daniels, S. R., Dishman, R. K., Gutin, B.... Trudeau, F. (2005). Evidenced based physcial activity for schoolage youth. Journal of Pedriatics, 146, 732-737.

Thorpe, K. E., Florence, C. S., Howard, D. H., \& Joski, P. (2004). The impact of obesity on rising medical spending. Health Affairs, Suppl Web Exclusives: W4-480-6

Troiano, R. P., Berrigan, D., Dodd, K. W., Masse, L. C., Tilert, T., \& McDowell, M. (2007). Physical activity in the United States measured by accelerometer Medicine \& Science in Sports \& Exercise, 40(1), 181-188.

Tudor-Locke C. E., \& Myers, A. M. (2001). Challenges and opportunities for measuring physical activity in sedentary adults. Sports Medicine, 31(2), 91100.

Vázquez, R., \& López, J. M. (2003). Los Costes de la Obesidad Alcanzan el 7\% del Gasto Sanitario. Recuperado de: http://www.economiadelasalud.com/Ediciones/03/PDF/03Analisis_Costes.pdf 